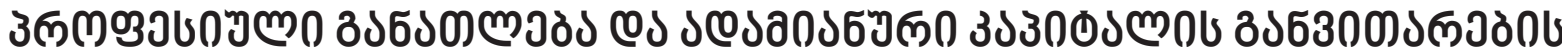

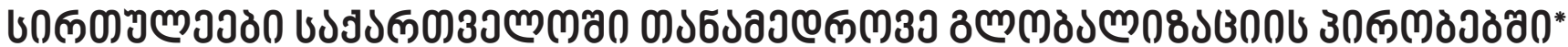

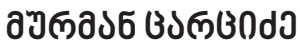

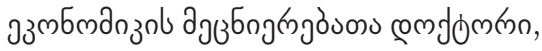

https://doi.org/10.35945/gb.2018.06.032

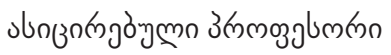

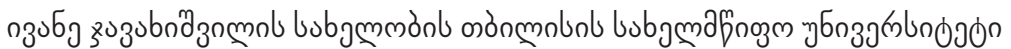

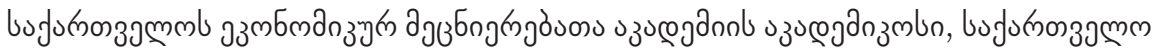

murman.tsartsidze@tsu.ge

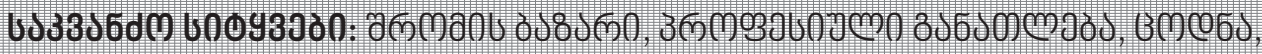

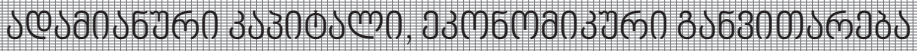

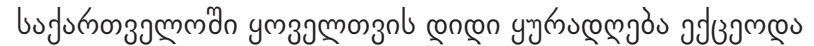

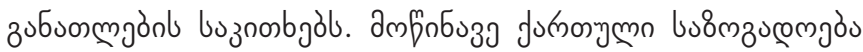

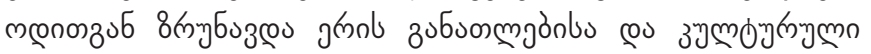

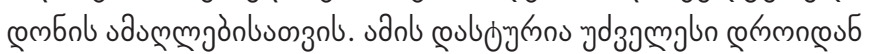

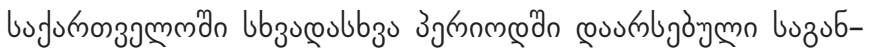

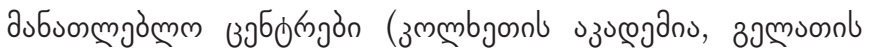

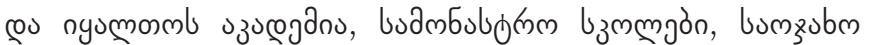

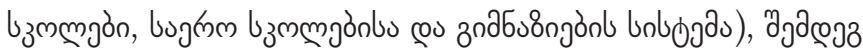

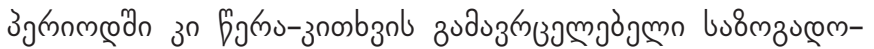

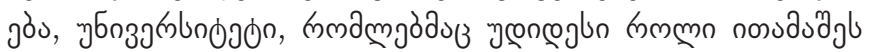

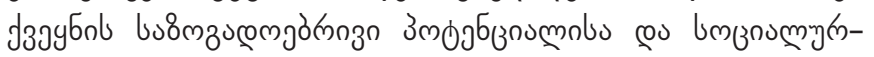

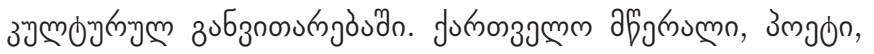

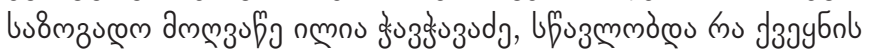

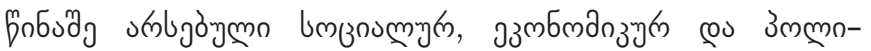

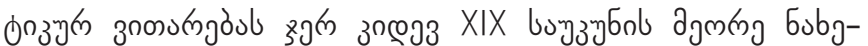

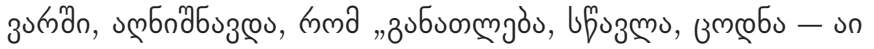

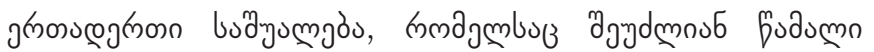

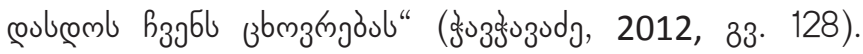

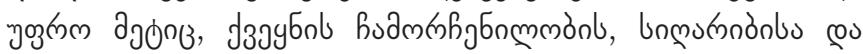

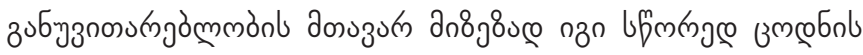

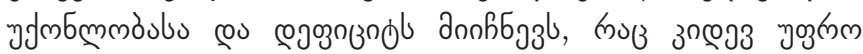

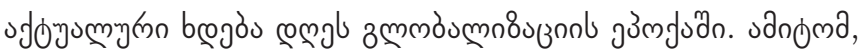

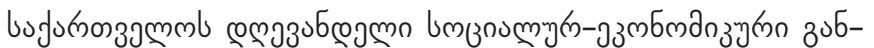

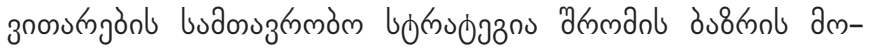

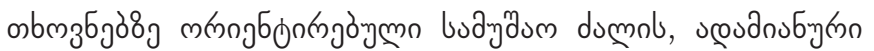

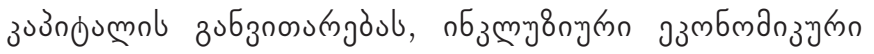

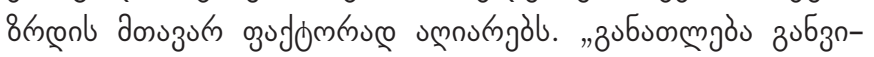

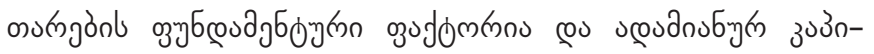

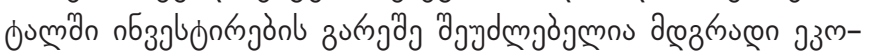

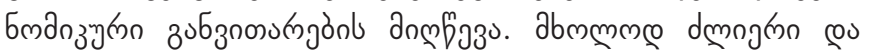

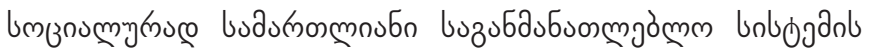

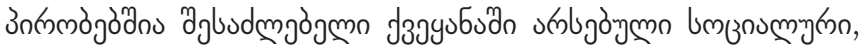

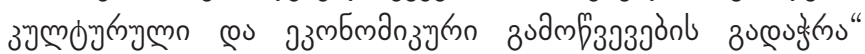

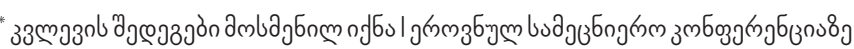

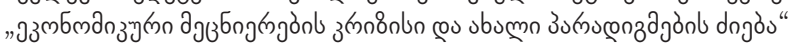

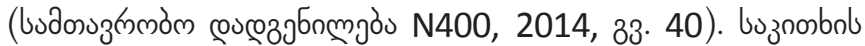

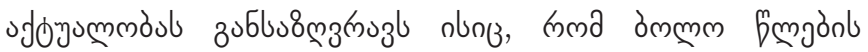

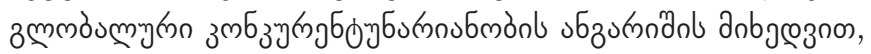

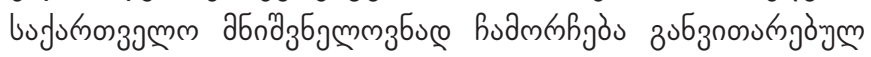

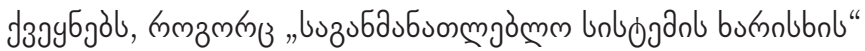

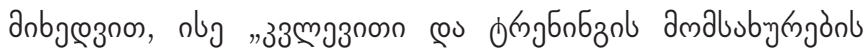

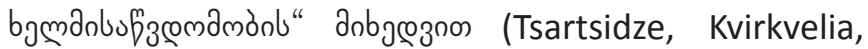

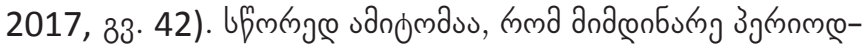

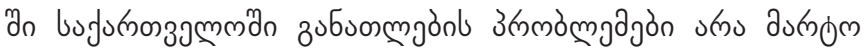

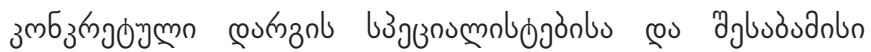

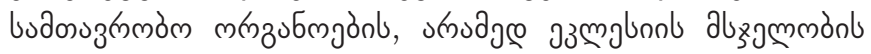

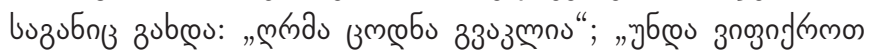

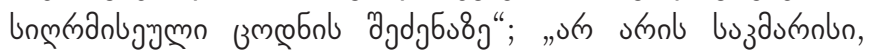

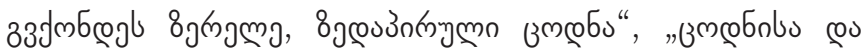

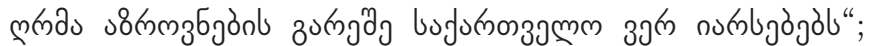

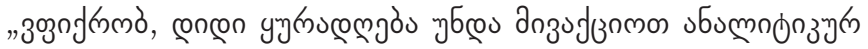

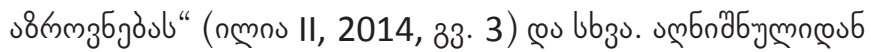

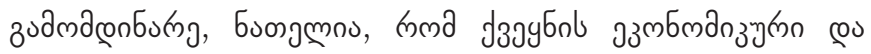

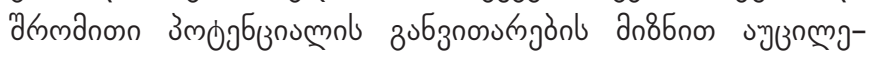

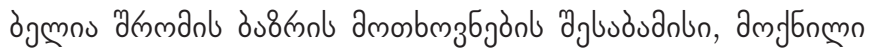

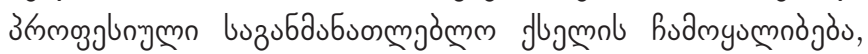

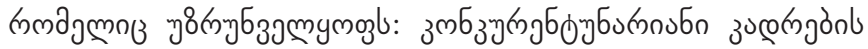

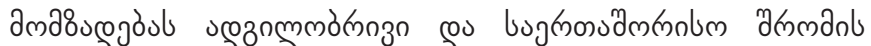

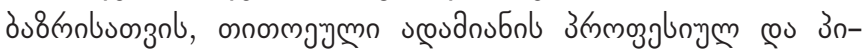
mмzб

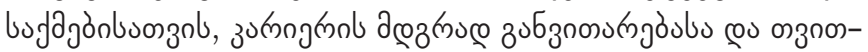

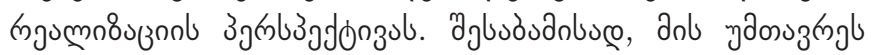

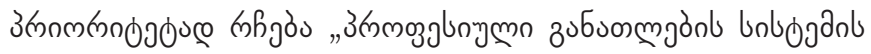

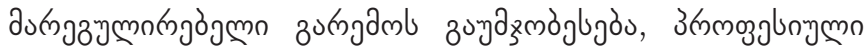

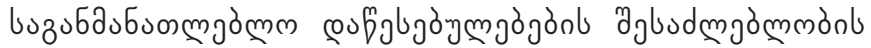

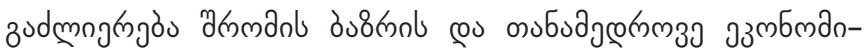

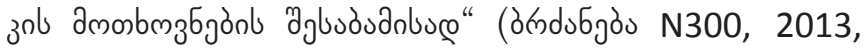

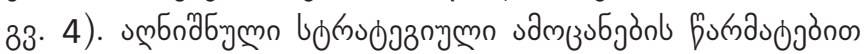

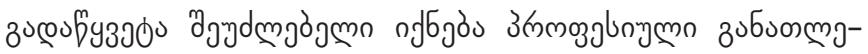

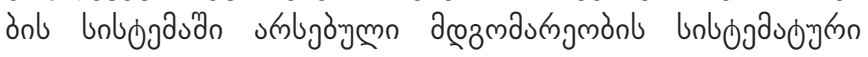

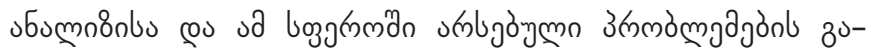


उbranga-1

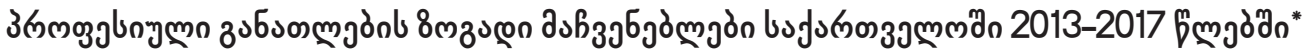

\begin{tabular}{|c|c|c|c|c|c|c|}
\hline \multicolumn{2}{|c|}{ 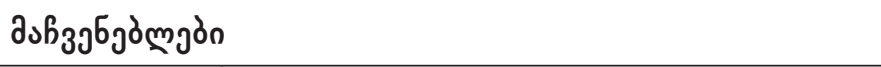 } & 2013 & 2014 & 2015 & 2016 & 2017 \\
\hline \multirow{8}{*}{ 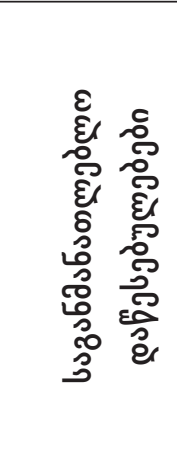 } & bygm & 104 & 140 & 140 & 120 & 125 \\
\hline & bozumm & 29 & 35 & 35 & 38 & 42 \\
\hline & 39 mdm & 75 & 105 & 105 & 82 & 83 \\
\hline & dso गmฒnl & & & & & \\
\hline & 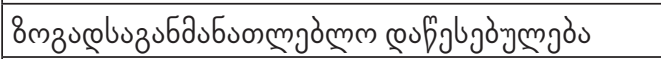 & 7 & 7 & 11 & 11 & 11 \\
\hline & 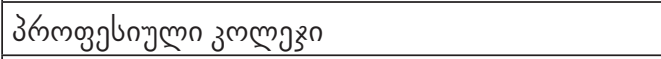 & 50 & 67 & 51 & 35 & 34 \\
\hline & 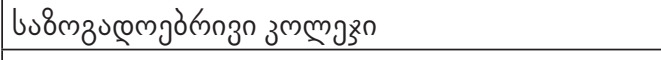 & 24 & 47 & 50 & 48 & 49 \\
\hline & 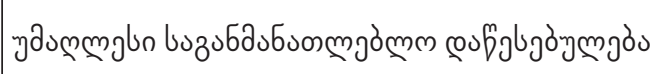 & 23 & 19 & 28 & 26 & 31 \\
\hline \multicolumn{2}{|c|}{ 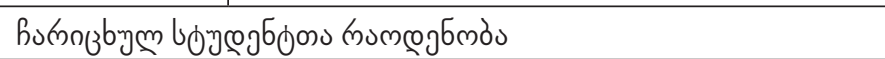 } & 21045 & 16182 & 16773 & 15639 & 15632 \\
\hline \multicolumn{2}{|c|}{ 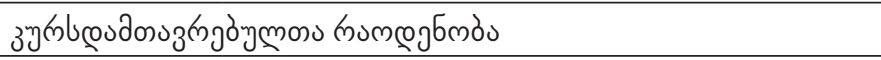 } & 10790 & 10810 & 11728 & 9943 & 9774 \\
\hline \multicolumn{2}{|c|}{ 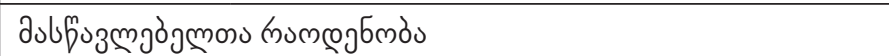 } & 2126 & 2808 & 3793 & 3642 & 4084 \\
\hline
\end{tabular}

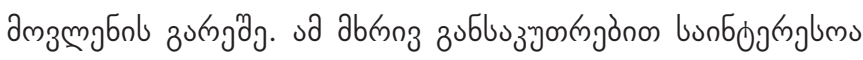

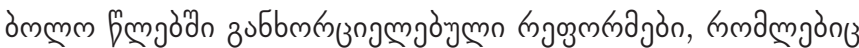

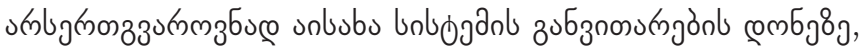

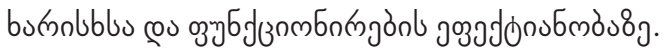

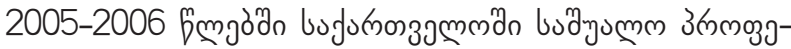

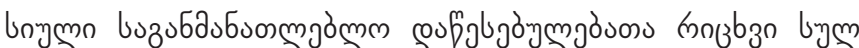

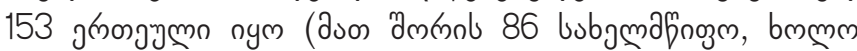

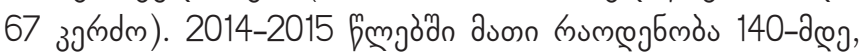

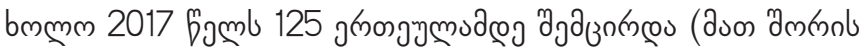

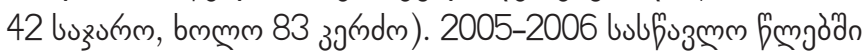

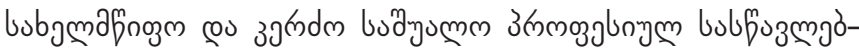

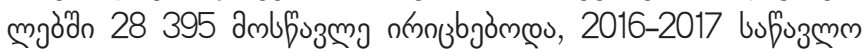

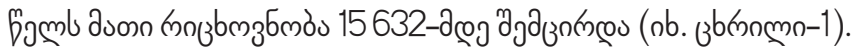

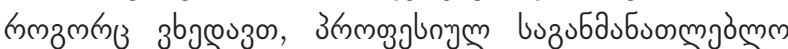

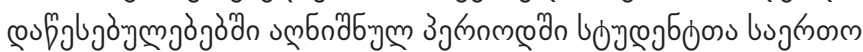

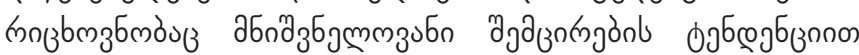

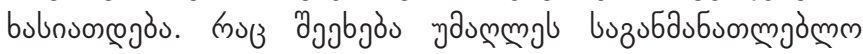

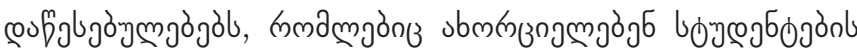

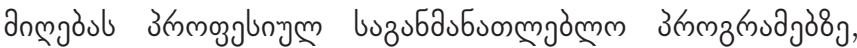

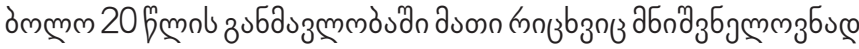

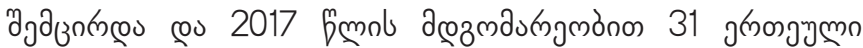

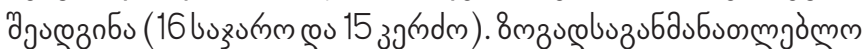

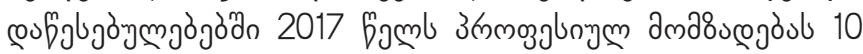

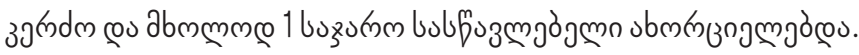

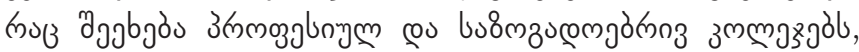

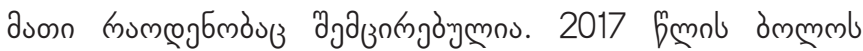

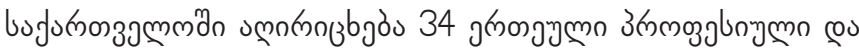

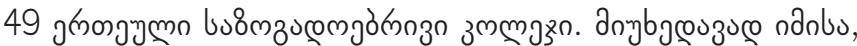

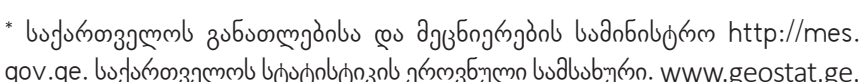

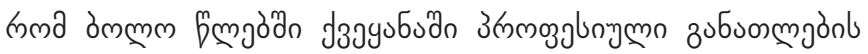

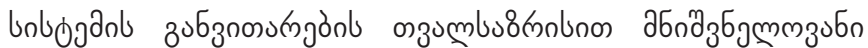

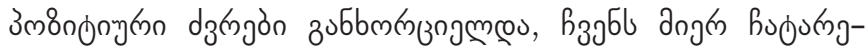

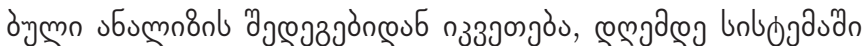
u

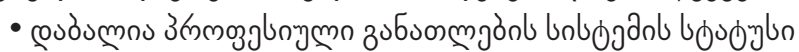

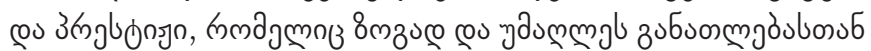

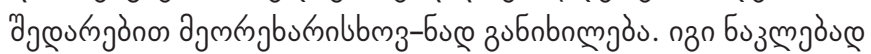
and8ge

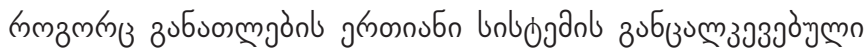

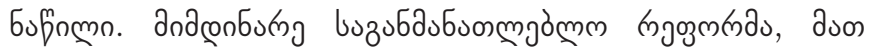

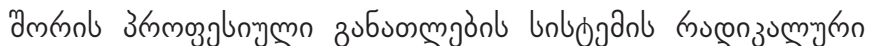

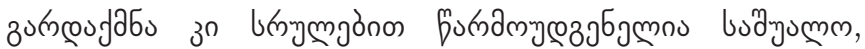

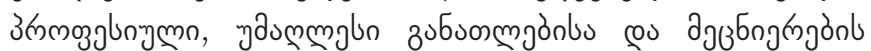

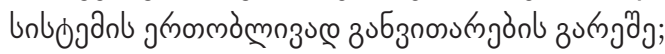

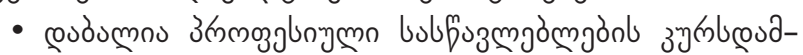

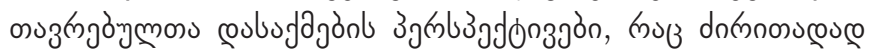

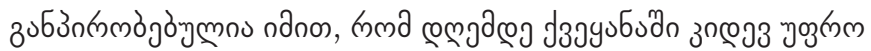

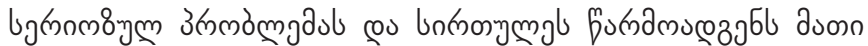

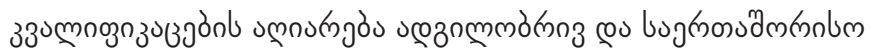

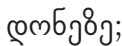

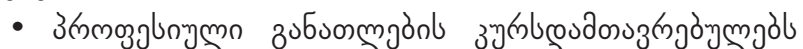

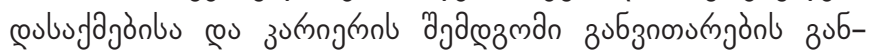

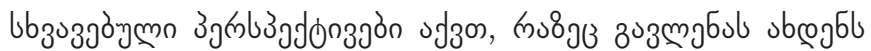

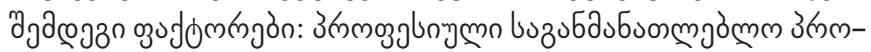

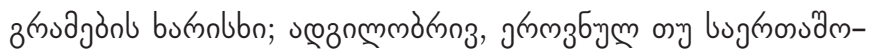

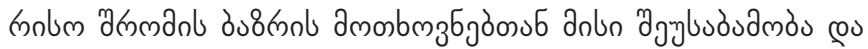

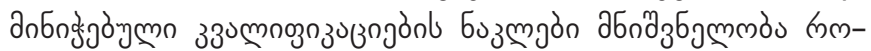

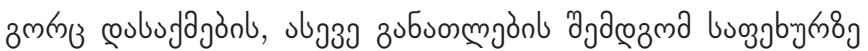

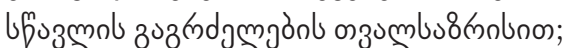

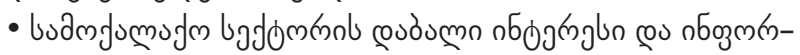




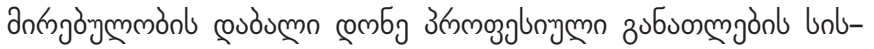

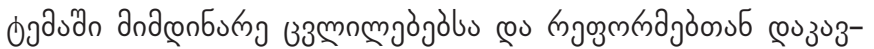

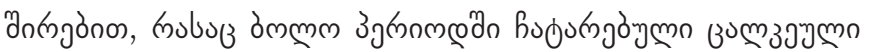

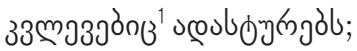

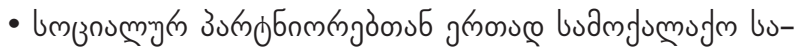

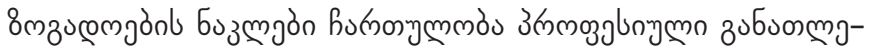

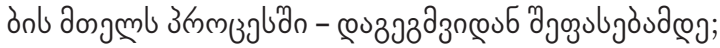

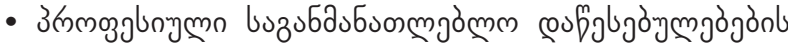

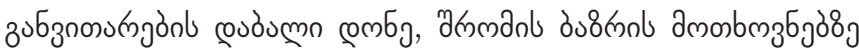

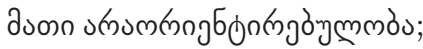

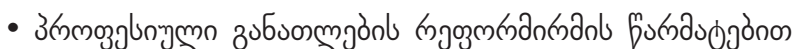

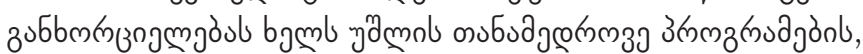

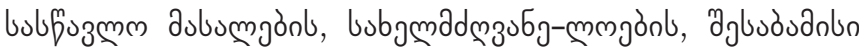

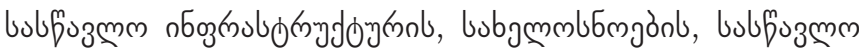

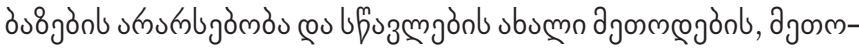

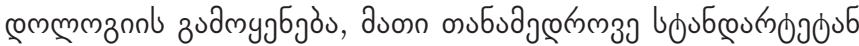

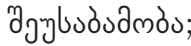

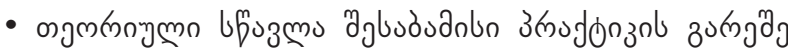

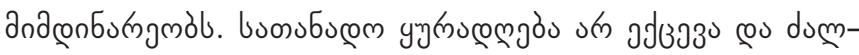

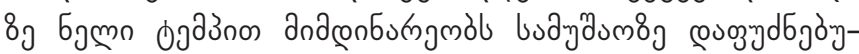

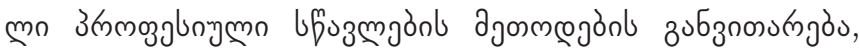

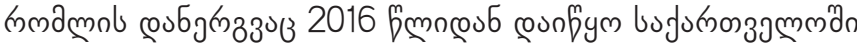

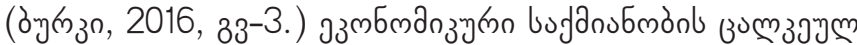
costrajogn;

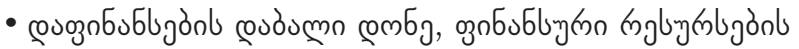

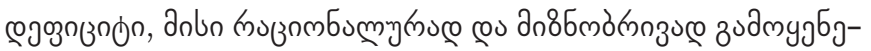

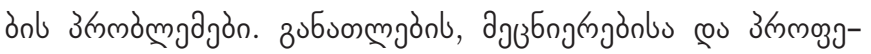

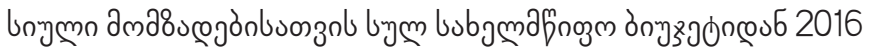

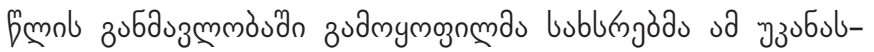

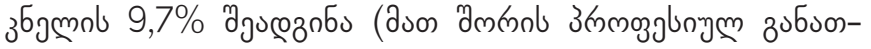
mృ

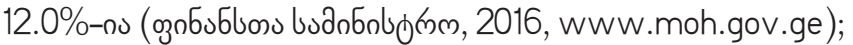

- bujunozzjmman 3

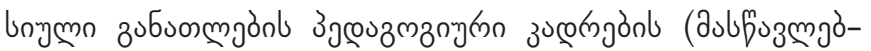

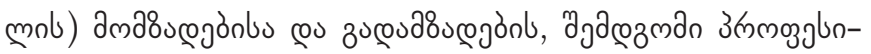

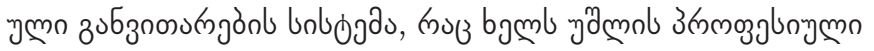

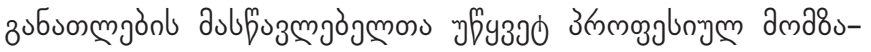

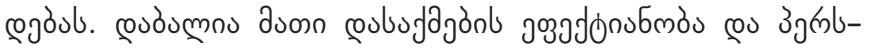

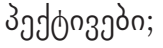

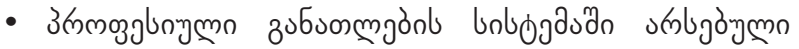

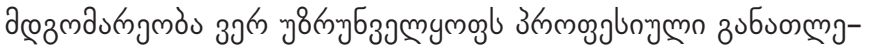

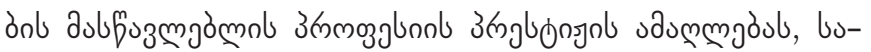

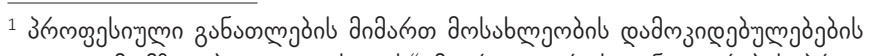

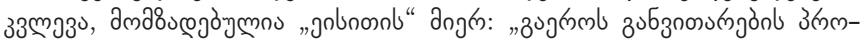

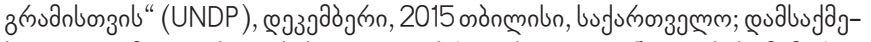

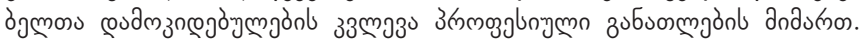

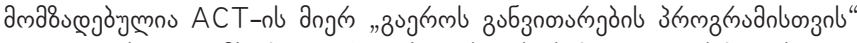

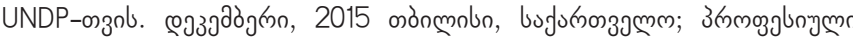

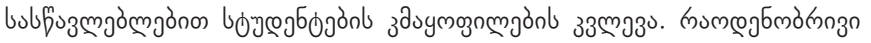

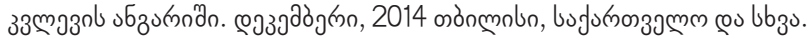

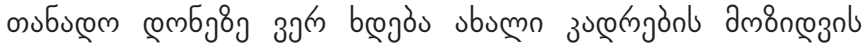

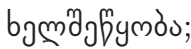

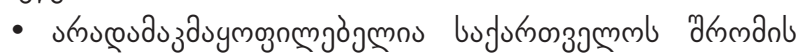

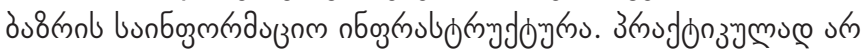

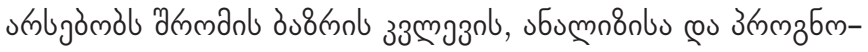

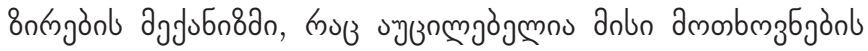

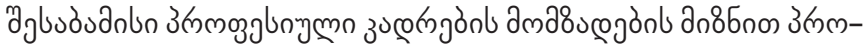

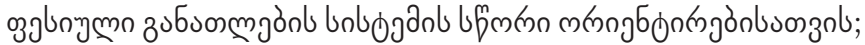

- b unbogan b a a cos bumnolibn;

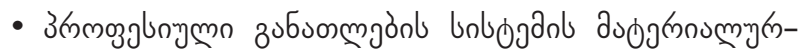

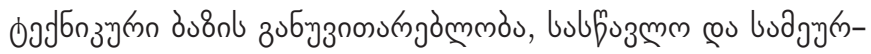
Бgm nб 356 бu

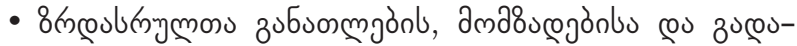

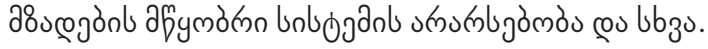

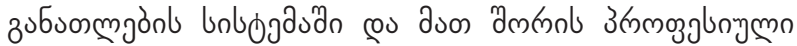

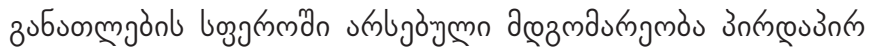
unbubjou u couanu

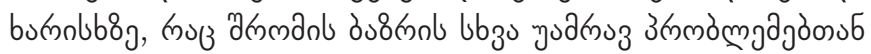

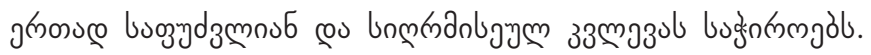

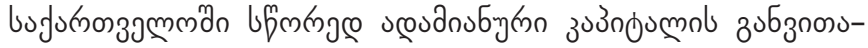

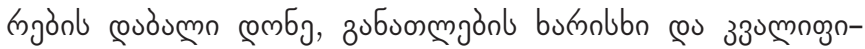

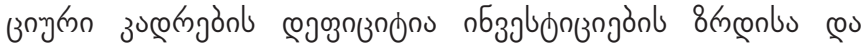

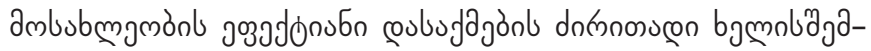

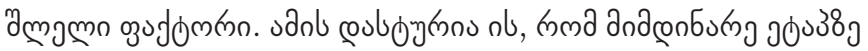

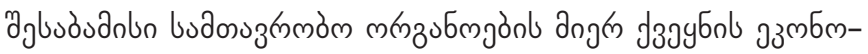

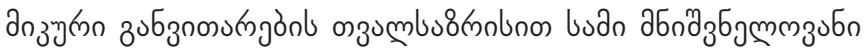
उஙnmòmgas zubnbng gòs:

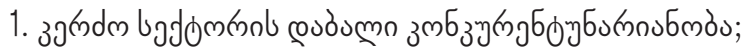

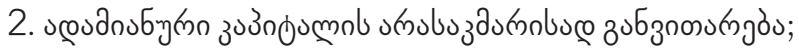

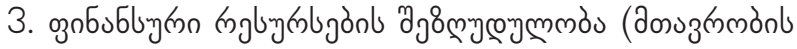

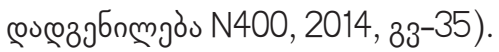

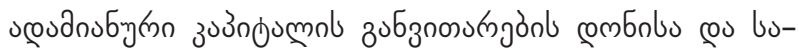

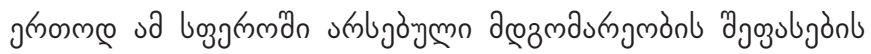

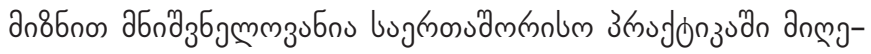

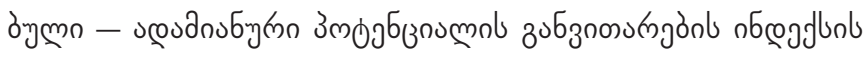
(Human Development Index trends/HDI/-UNDP.HDI: http://

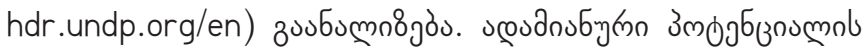

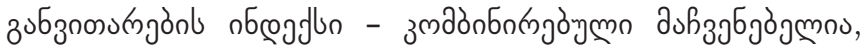

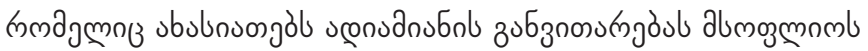

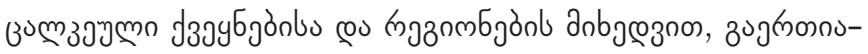

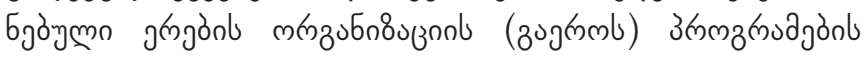

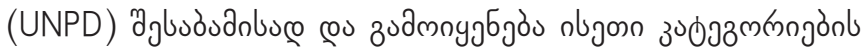

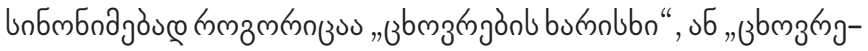
on combु".

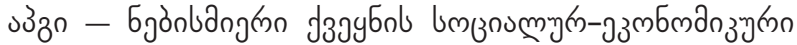

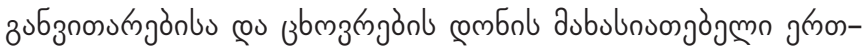

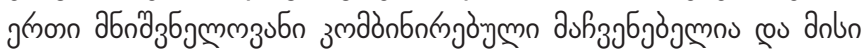




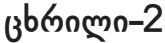

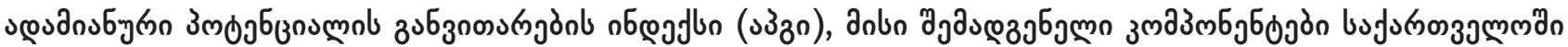

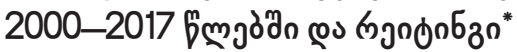

\begin{tabular}{|c|c|c|c|c|c|c|}
\hline & 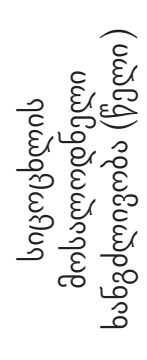 & 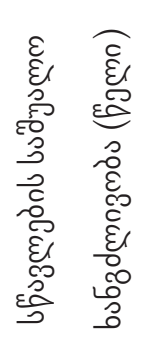 & 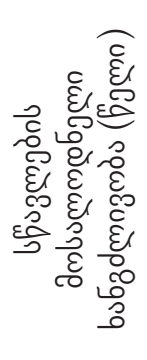 & 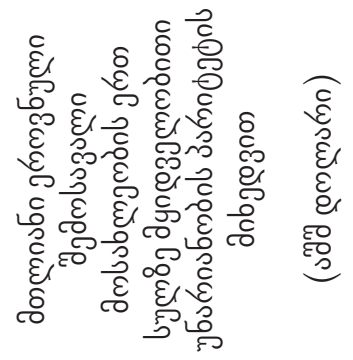 & 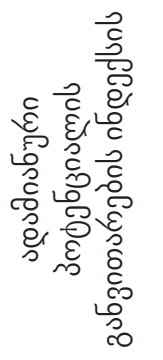 & 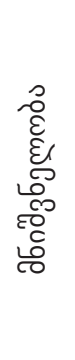 \\
\hline 2000 & 71,8 & 11,7 & 11,7 & 3407 & 0,673 & \\
\hline 2005 & 73,2 & 12,6 & 12,1 & 5027 & 0,714 & \\
\hline 2010 & 74,2 & 13,3 & 12,2 & 6530 & 0,742 & \\
\hline 2011 & 74,4 & 13,4 & 12,1 & 7101 & 0,749 & \\
\hline 2012 & 74,6 & 13,5 & 12,2 & 7810 & 0,755 & \\
\hline 2013 & 74,7 & 13,5 & 12,2 & 8098 & 0,759 & \\
\hline 2014 & 74,9 & 13,9 & 12,2 & 8670 & 0,768 & \\
\hline 2015 & 75,0 & 13,9 & 12,2 & 8856 & 0,769 & \\
\hline 2016 & 72,7 & 13,9 & 12,2 & 9410 & 0,777 & \\
\hline 2017 & 73.5 & 13,9 & 12,2 & 9435 & 0.782 & \\
\hline
\end{tabular}

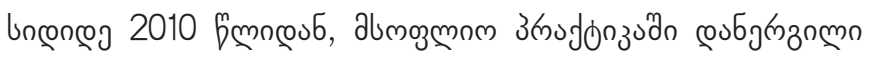

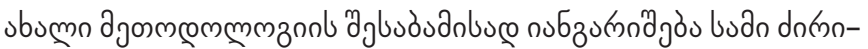

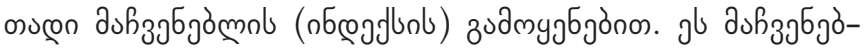
mgòno:

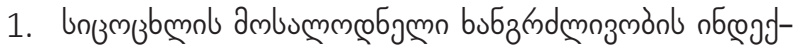

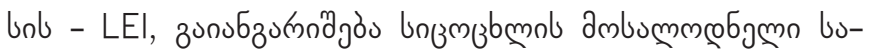

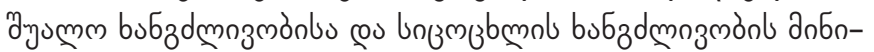
aumingn 8

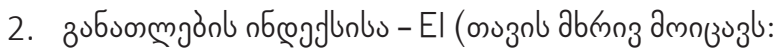

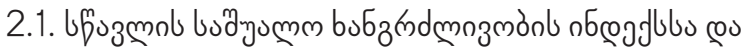

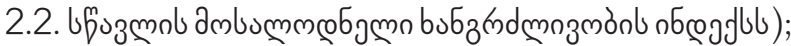

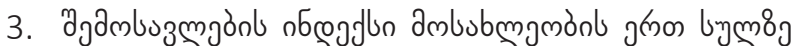

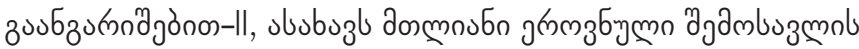

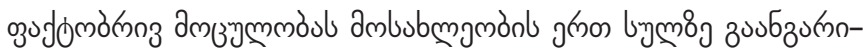

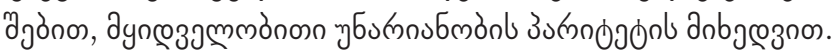

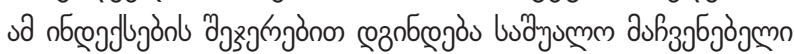

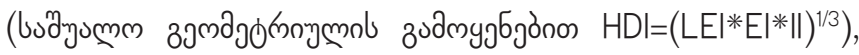

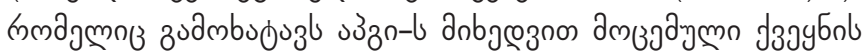

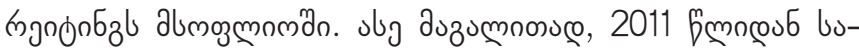

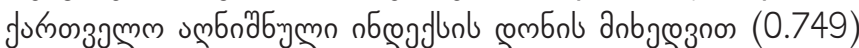

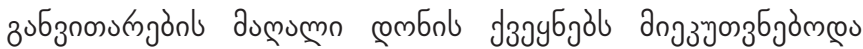

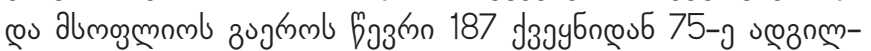

* United Nations Development Programme. Human Development Reports. International Human Development Indicators. Gorgia. http://hdr.undp.org/en/countries; Human Development Report 2016. http://hdr.undp.org/sites/all/themes/hdr-theme/countrynotes/GEO.pdf.Top of Form

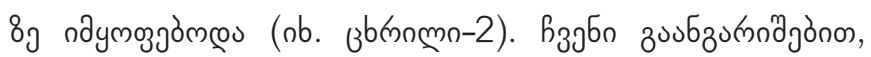

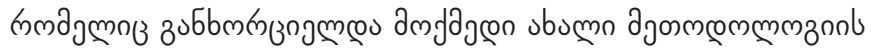

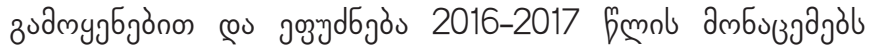

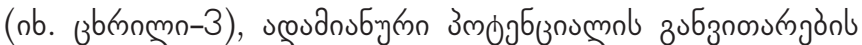

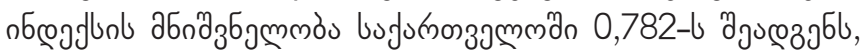

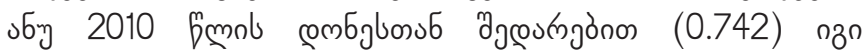

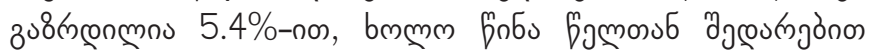

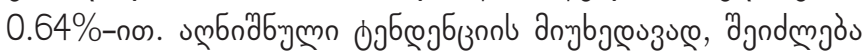

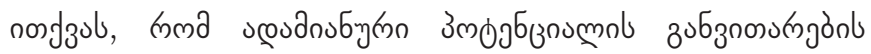

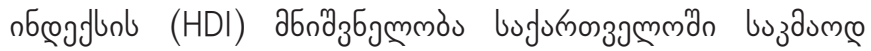

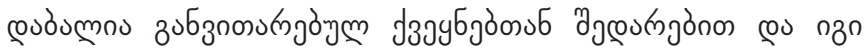

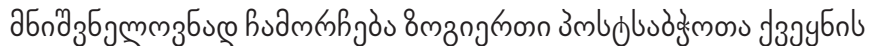

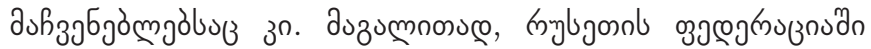

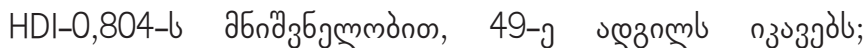

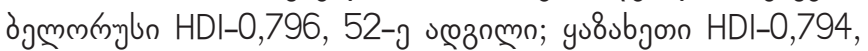

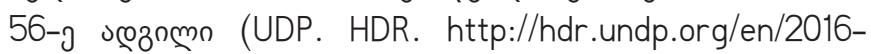

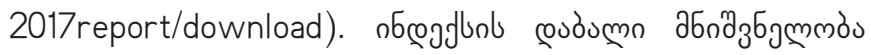

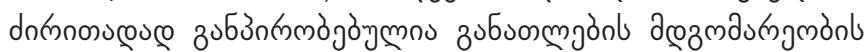

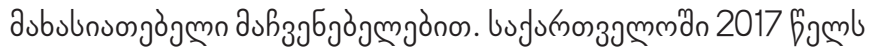

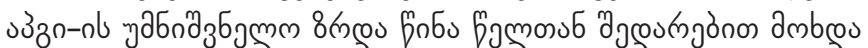

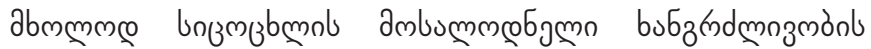

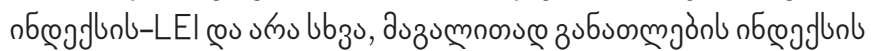

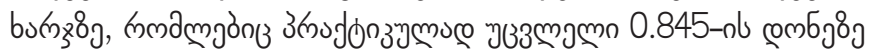
coumbo.

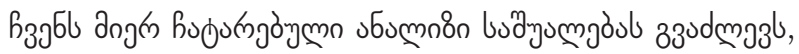

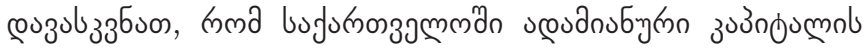

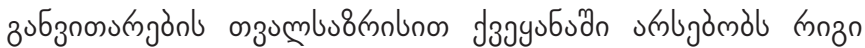




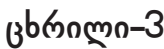

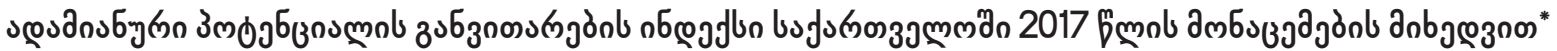

\begin{tabular}{|c|c|c|c|}
\hline 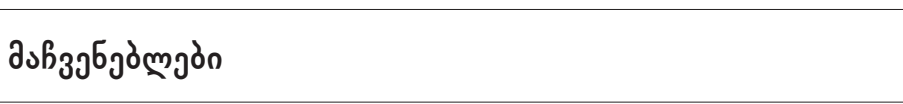 & 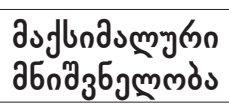 & 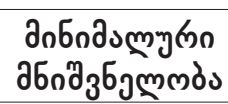 & 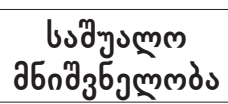 \\
\hline 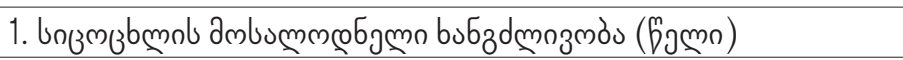 & 85 & 20 & 73.5 \\
\hline 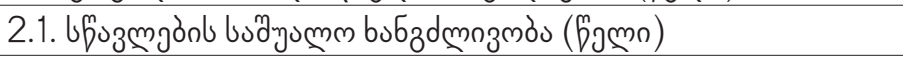 & 13.3 & 0 & 12.2 \\
\hline 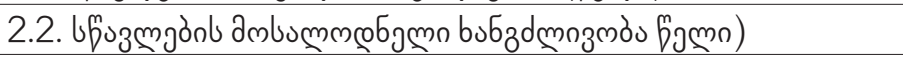 & 18 & 0 & 13.9 \\
\hline 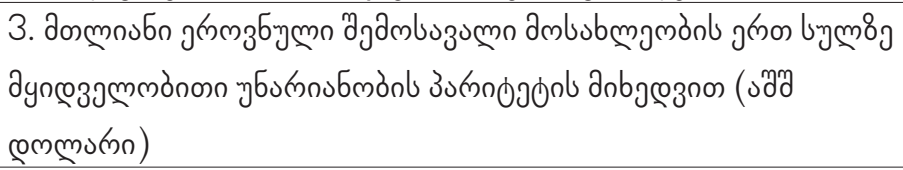 & 75000 & 100 & \\
\hline \multirow{3}{*}{$\mathrm{HDI}=(\mathrm{LEI} * \mathrm{EI} * \mathrm{II})^{1 / 3}=\underline{0.782}$} & \multicolumn{2}{|c|}{$L E I=\left(L E-L E_{\partial 06}\right) /\left(L_{\partial_{000}}-L E_{\partial 06}\right)$} & 0.823 \\
\hline & \multicolumn{2}{|c|}{ 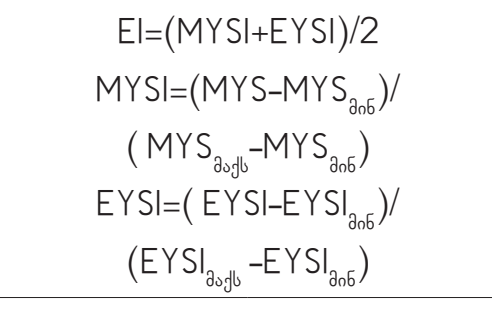 } & 0.845 \\
\hline & \multicolumn{2}{|c|}{$\begin{array}{l}\|=(\ln (G N \mid p c)-\ln (100)) / \\
(\operatorname{lnGNI}(75000)-\ln (100)\end{array}$} & 0.687 \\
\hline
\end{tabular}

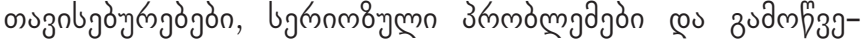

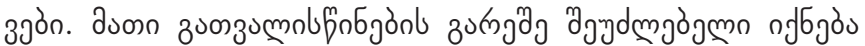

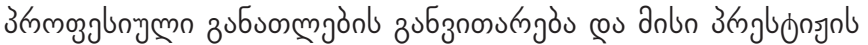

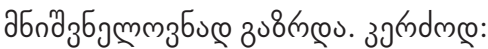

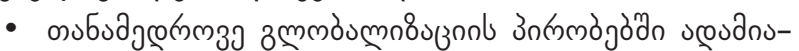

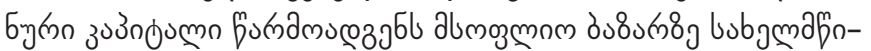

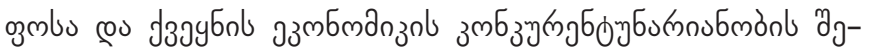

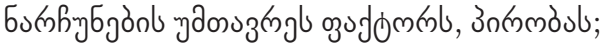

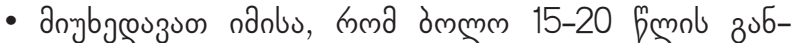

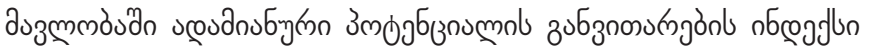

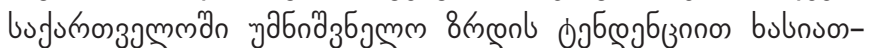

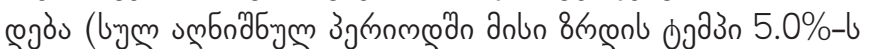

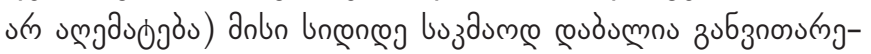

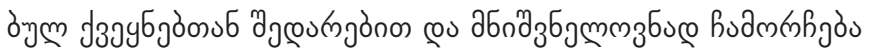

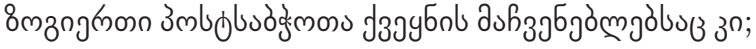

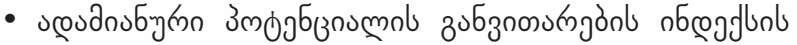

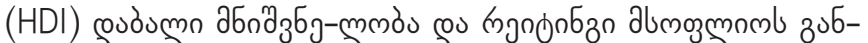

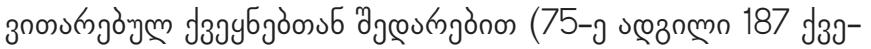

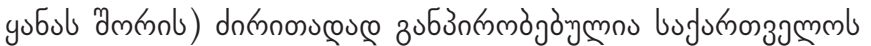

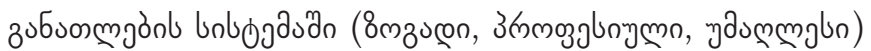
ง

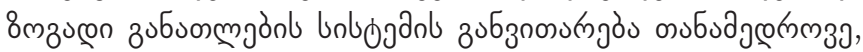

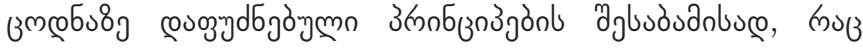

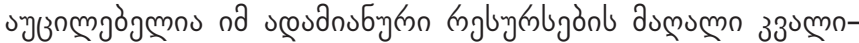

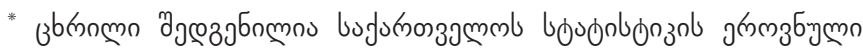

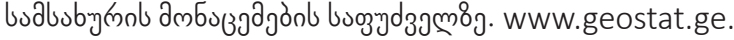

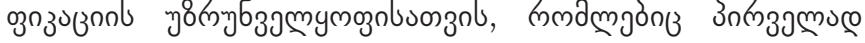

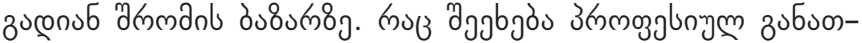

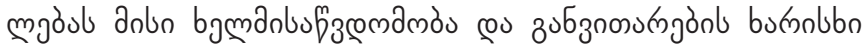

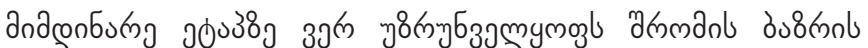
amonbmzбu\&

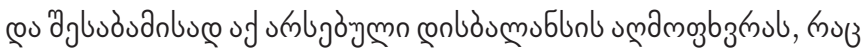

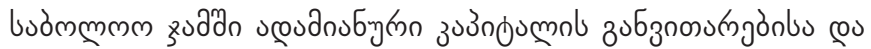

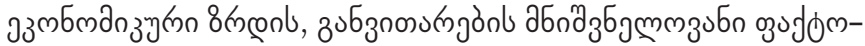

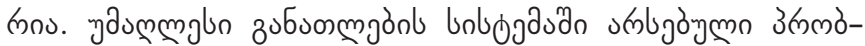

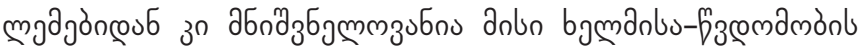

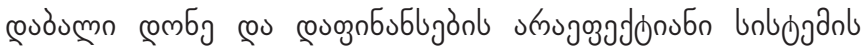
smbjòmós;

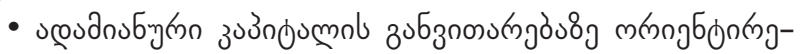

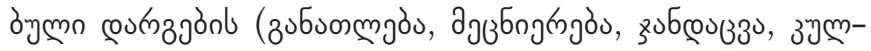

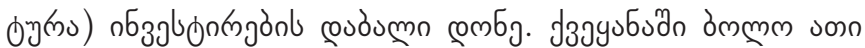

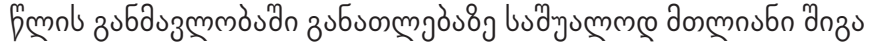

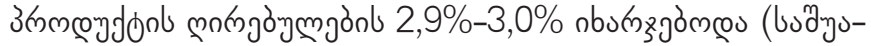

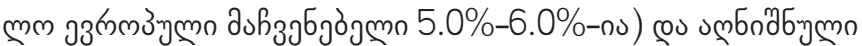

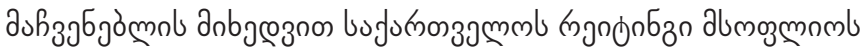

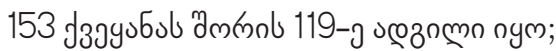

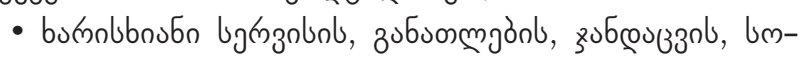

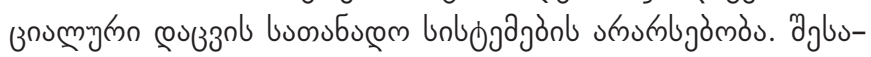

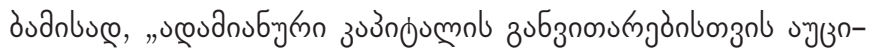

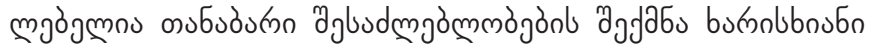

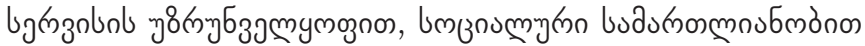

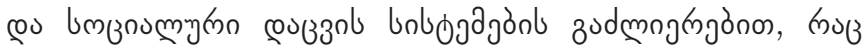

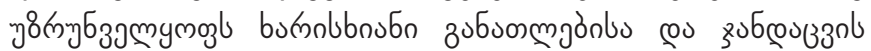




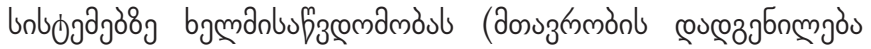
N400, 2014, 33. 35)";

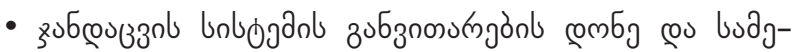

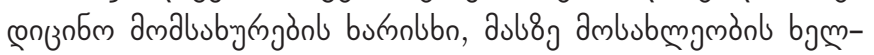
anbufzsemamòn zu8knos;

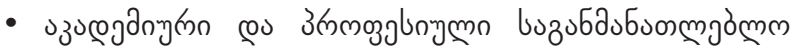

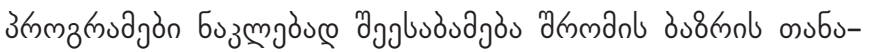

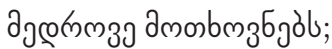

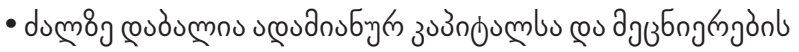

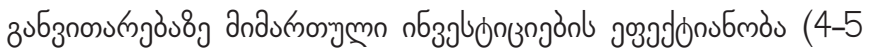

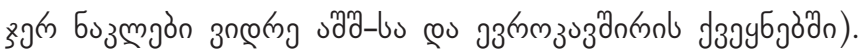

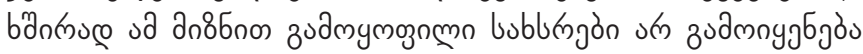

उ०œ

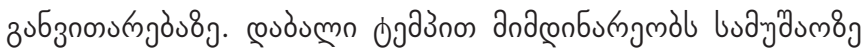
cougyd

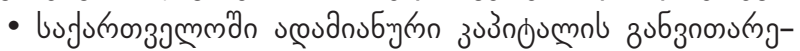
onb ozumbus

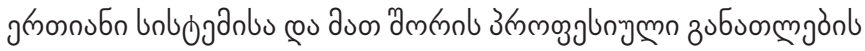

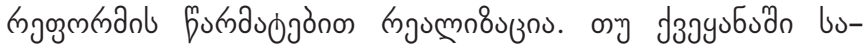

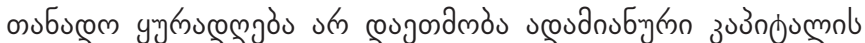

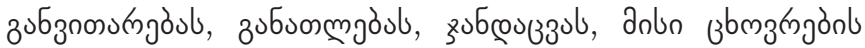

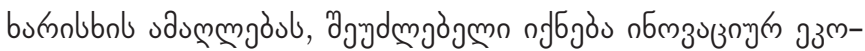

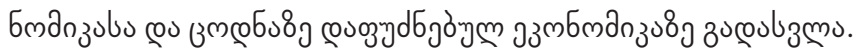

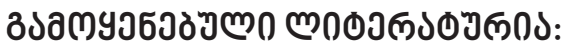

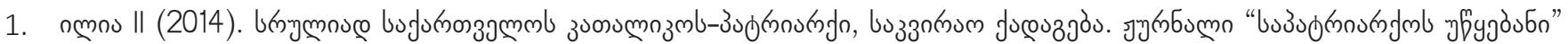

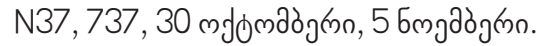

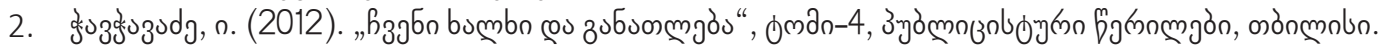

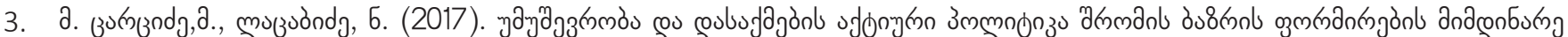

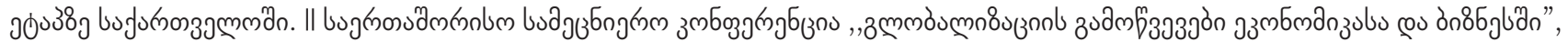

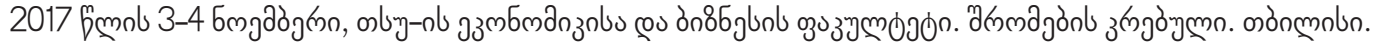

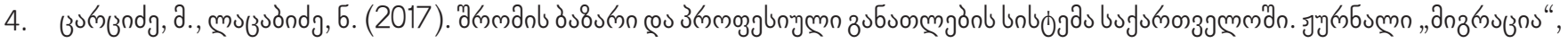

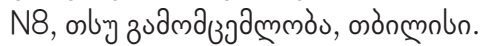

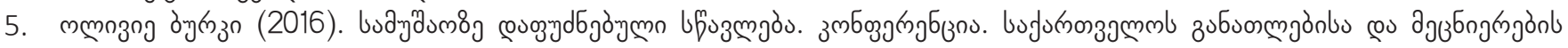

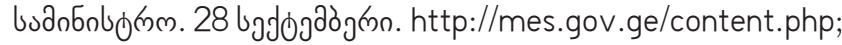

6. Tukhashvili, M. (2012). Georgian Labour Market in the condition of Political and Economic Crisis. In the book:Youth Employment: Challenges and Opportunites. Timisoara. Eurostampa.

7. Murman Tsartsidze, M., Kvirkvelia, E. (2017). Labor market Oriented Professional Education System - the Significant Factor for Human Capital Development and the Inclusive Economic Growth in Georgia. Journal of International Economic Research, ISSN 2500-9656. Volume 3. p.21-28. http://irissymposium. wixsite.com/economics/proceedings.

8. Tsartsidze, M. (2016). Poverty and the Economic Development Factors in Georgia. «European Journal of Humanities and Social Sciences», Scientific journal, №1, vienna.

9. Tsartsidze, M. (2013). Labour Market Regulation and Employment Policies in Georgia. European Applied Scinces N2/2, 2013. stuttgard, Germany.

10. United Nations Development Programme. Human Development Reports. http://hdr.undp.org/en/2016-2017report/ download.

11. United Nations Development Programme. Human Development Reports. International Human Development Indicators. Gorgia. http://hdr.undp.org/en/countries;

12. Human Development Report 2016.http://hdr.undp.org/sites/all/themes/hdr-theme/country-notes/GEO.pdf.

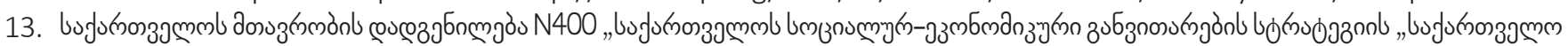

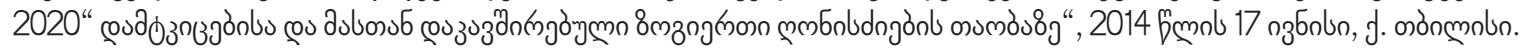

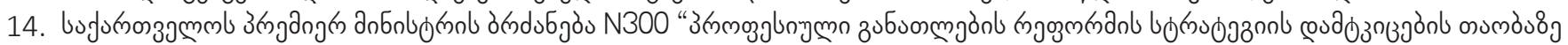

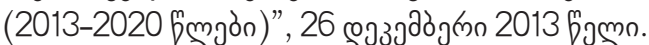

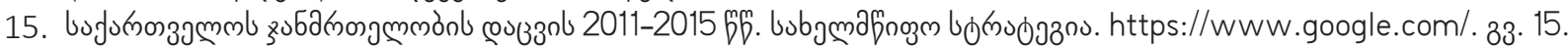

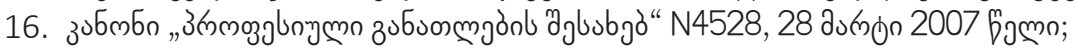

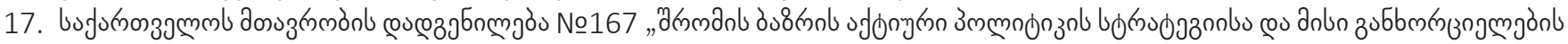

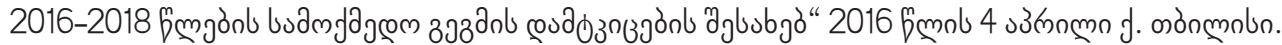

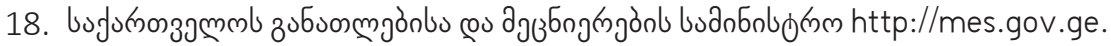

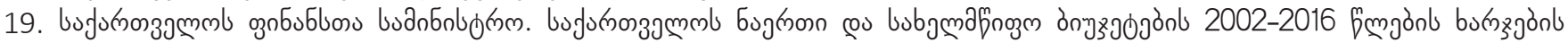

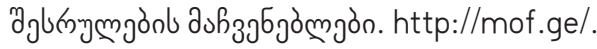

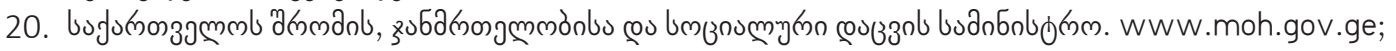

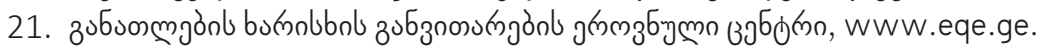

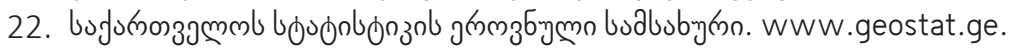




\section{PROFESSIONAL EDUCATION AND HUMAN CAPITAL DEVELOPMENT DIFFICULTIES IN GEORGIA UNDER THE MODERN GLOBALIZATION TERMS}

\section{MURMAN TSARTSIDZE}

https://doi.org/10.35945/gb.2018.06.032

Doctor of Economic Sciences, Associate professor, Ivane Javakhishvili Tbilisi State University,

Academician of the Academy of Economic Sciences of Georgia, Georgia

murman.tsartsidze@tsu.ge

"Education, Study, knowledge - the only remedy in our lives"

\section{Ilia Chavchavadze}

"It is necessary for us to think about acquiring the deep knowledge, and that without knowledge and deep thinking Georgia will not survive"

Ilia the II

\section{KEYWORDS: LABOR MARKET, PROFESSIONAL EDUCATION, KNOWLEDGE} HUMAN CAPITAL, ECONOMIC DEVELOPMENT

\section{SUMMARY}

Unfortunately, in the epoch of modern globalization, the main priority of the country is still the unemployment and the poverty overcoming. In such situation it is clear, that the economic development and the significant improvement of the population's living standards cannot be achieved without human resources development, effective use of the labor potential, formation of civilized labor market and organization of the professional educational system according to the modern standards. The latter should favor the development of the main state strategy task - labor force oriented to the labor market demands and accordingly, the human capital develop- ment, significant increase of the competitiveness in the local and international markets.

Proceeding from the actuality of the issue, in the work the role of education, especially the professional education, as the social-cultural phenomena is considered. The main problems and challenges of the field are studied. We have documented that in the terms of modern globalization the level, quality of functioning of the professional educational system and its correct orientation towards the labor market demands to determine the human capital development in the country, labor market potential their quality perfection and their use prospective. 